\title{
Der Einsatz digitaler Medien an der Grundschule. Eindrücke aus dem Projekt dileg-SL aus der Per- spektive von Lehrkräften.
}

\author{
Thorsten Junge
}

\begin{abstract}
Zusammenfassung des Beitrags
Im Rahmen des Entwicklungsprojekts „Digitales Lernen Grundschule - Stuttgart/Ludwigsburg“ (kurz: dileg-SL) haben Lehramtsstudierende der Pädagogischen Hochschule Ludwigsburg verschiedene Konzepte für eine produktive Nutzung digitaler Medien im Grundschulunterricht erstellt. Diese wurden in Kooperation mit den Lehrkräften der Rosensteinschule in Stuttgart mit verschiedenen Grundschulklassen erprobt. In dem vorliegenden Beitrag stehen die Eindrücke der mitwirkenden Lehrer*innen im Fokus. Hierzu wurden fünf Lehrkräfte in leitfadengestützten Interviews befragt. Die Auswertung der Gespräche zeigt u. a., welche Potentiale die Lehrer*innen beim Einsatz digitaler Medien sehen und welche Hindernisse bei der (nachhaltigen) Implementierung im Grundschulalltag bestehen.
\end{abstract}

Schlüsselbegriffe: Medienpädagogik $\bullet$ PH Ludwigsburg •Projekt dileg-SL $\bullet$ Grundschule $\bullet$ Digitale Medien $\bullet$ Lebrerbefragung

\section{Einleitung: digitale Medien in der Schule}

Die zunehmende Durchdringung aller gesellschaftlichen Bereiche mit digitalen Medien stellt auch die Schule vor verschiedene Herausforderungen. Als Reaktion auf die gesellschaftlichen Entwicklungsprozesse wurde von den politischen Entscheidungsträgern u. a. in den Empfehlungen der Kultusministerkonferenz (KMK) festgehalten, dass bereits für Kinder „Bildung in der digitalen Welt" (KMK 2016) möglich sein soll und Schüler*innen sollen bei der Entwicklung „Digitaler Souveränität“ unterstützt werden (Aktionsrat Bildung 2018). Aus medienpädagogischer Sicht können diese Forderungen erfüllt werden, wenn digitale Medien in der Schule nicht nur thematisiert, sondern auch eingesetzt werden.

Die Frage, ob und in welchem Ausmaß digitale Medien in der Schule zum Einsatz kommen können, wurde in den letzten Jahren in der Schul- und Medienpädagogik intensiv diskutiert, wobei sich (euphorische) Befürworter und (weniger technikaffine) Skeptiker gegenüberstehen (vgl. Irion/Scheiter 2018). Mit Blick auf die Schulformen scheint es hierbei verschiedene Argumentationslinien zu geben. Während die zielführende Verwendung in den weiterführenden Schulformen (und hier insbesondere an den Gymnasien) nach meiner Wahrnehmung deutlich 
mehr Befürworter findet, wurde die Eignung für die Grundschule lange Zeit in Zweifel gezogen, auch wenn nach Einschätzung der KMK „das Lernen mit und überdigitale Medien und Werkzeuge bereits in den Schulen der Primarstufe beginnen" (KMK 2016, S. 11) sollte. Das junge und schutzbedürftige Alter der Grundschüler*innen sowie die steigende Bedeutung im privaten Alltag der Kinder (u. a. abzulesen anhand der Daten der KIM-Studien; vgl. MPFS 2020) dient mitunter als Argument, um einen „medienfreien Schonraum“ zu propagieren (vgl. Neuss/Spitzer 2012). Und in den meisten Bundesländern wurde bis dato noch nicht konkretisiert, wie „die KMK-Dimensionen für die Grundschule adaptiert werden“ (Kammerl et al. 2020, S. 35). Demgegenüber hat sich der Grundschulverband in einer Stellungnahme positioniert und die Förderung der „digitalen Mündigkeit“ angemahnt (Grundschulverband 2018) und die Formulierung spezifischer Grundschulstandards gefordert. Hierbei sollte berücksichtigt werden, „dass die Schülerschaft an Grundschulen meist sehr heterogen zusammengesetzt ist und aus unterschiedlichen Sozialisationskontexten kommt." (Niesyto 2020, S. 192)

Gerade die Grundschule ist ein überaus geeigneter Ort, um den sozialen Ungleichheiten etwas entgegenzusetzen, die durch die private Mediennutzung entstehen (vgl. zum Thema digitale Ungleichheit u.a. Niesyto 2019a; vgl. ausführlich zum Thema „Digitale Bildung ${ }^{1}$ im Grundschulalter" Thumel/Kammerl/Irion 2020). So haben Studien wie der 14. Kinder- und Jugendbericht Unterschiede in den Nutzungsweisen aufgezeigt und insbesondere Kinder aus bildungsfernen Milieus nutzen die Potenziale der digitalen Online-Medien deutlich weniger zum Wissenserwerb (vgl. Bundesministerium für Familie, Senioren, Frauen und Jugend 2013). Im Fachunterricht sowie in außerunterrichtlichen Lernangeboten (z. B. AGs oder Projektwochen) sollte man bereits Grundschüler*innen die grundlegenden Fähigkeiten für ein sachgerechtes, selbstbestimmtes, kreatives und sozial verantwortliches Medienhandeln vermitteln (vgl. Tulodziecki/Herzig/Grafe 2010, S. 70 f.). Dabei scheinen insbesondere Tablets gut geeignet zu sein: „Als flexibel und schnell einsetzbare Multimediageräte mit vielfältigen Apps und der Integration von Kamera und Mikrophon bieten sich vielseitige Einsatzmöglichkeiten zur Anbahnung von Medienkompetenzen und Gestaltung von Lernprozessen in der Schule“ (Tillmann 2018, S. 13).

Zusätzlich zu den skeptischen Positionen gegenüber dem Einsatz digitaler Medien in der Grundschule erschwert die unzureichende technische Ausstattung an vielen Schulen deren Implementierung (vgl. Bitkom 2019; Zylka 2018, S. 25). Im internationalen Vergleich sind deutsche Schulen deutlich schlechter ausgestattet als Schulen in anderen Ländern (vgl. Eickelmann et al. 2019; Zylka 2018, S. 31). Hier besteht die Hoffnung, dass die Maßnahmen im Zuge des „DigitalPakt Schule“ (BMBF 2019) zukünftig die Verfügbarkeit der technischen Ressourcen zur Förderung der Medienbildung verbessern (vgl. Aufenanger 2020a, S. 7), sodass der Einsatz digitaler Medien im Unterricht und in Projektkontexten erleichtert wird. Mit der finanziellen Förderung wirkt der Bund gewissermaßen als „Katalysator“ (Knaus 2019, S. 18) für die notwendigen Veränderungsprozesse und versucht auf diese Weise, „den Knoten [zu] lösen, der durch das gegenseitige Zuschieben von Verantwortung [zwischen Ländern, Kommunen und Kreisen] über die Ausstattung mit digitalen Medien vielerorts entstanden ist" (Knaus

\footnotetext{
${ }^{1}$ An dieser Stelle soll ein kurzer Hinweis genügen, dass die Bezeichnung „digitale Bildung“ auch kritisch gesehen wird (vgl. hierzu Niesyto/Junge 2020, S. 316).
} 
2019, S. 18) Allerdings deutet sich bereits an, dass weniger Mittel an den Grundschulen investiert werden als an weiterführenden Schulen (vgl. Niesyto 2020, S. 208).

Vor diesem Hintergrund erscheint es sinnvoll, das kreative Potential von Medien für Bildungs- und Lernprozesse an der Grundschule mithilfe von handlungsorientierten Projekten aufzuzeigen. Denn neben einer verlässlichen Infrastruktur mit digitalen (Online-)Medien ist vor allem eine wohlwollende Einstellung der Lehrer*innen für die Implementierung digitaler Medien an Grundschulen von Bedeutung (vgl. u.a. Aufenanger 2020b). Und diese lässt sich leichter herstellen, wenn sie den Mehrwert digitaler Medien konkret erfahren können.

\section{Das Projekt dileg-SL ${ }^{2}$}

An der Pädagogischen Hochschule Ludwigsburg fand von April 2016 bis September 2019 das Entwicklungsprojekt „Digitales Lernen Grundschule - Stuttgart/Ludwigsburg“ (kurz: dilegSL) statt (vgl. Junge/Niesyto 2019). Das praxisorientierte Projekt wurde im Rahmen des Förderprogramms „Digitales Lernen Grundschule“ der Deutsche Telekom Stiftung gefördert und gehörte zu einem Verbundprojekt, welches an fünf weiteren Standorten in Deutschland durchgeführt wurde. Kooperationspartner war die Rosensteinschule in Stuttgart.

Die Rosensteinschule ${ }^{3}$ ist eine Grund- und Werkrealschule (teilgebundene Ganztagsschule) und liegt in einem multikulturell geprägten Bezirk der Stuttgarter Innenstadt (Nordbahnhofviertel). Dies hat auch Einfluss auf die Zusammensetzung der Schülerschaft: Der Anteil der Schüler*innen mit Migrationshintergrund liegt bei über 90 Prozent. Folgt man dem Sozialdatenatlas der Stadt Stuttgart ist der Anteil von einkommensschwachen und sogenannten bildungsfernen Eltern im Einzugsgebiet der Rosensteinschule überproportional hoch. Insgesamt besuchen etwa 200 Schüler*innen die Grundschule.

Im Entwicklungsprojekt dileg-SL stand die Frage im Vordergrund, wie digitale Medien zielführend in der Grundschule eingesetzt werden und wie damit einhergehend (angehende) Lehrer*innen im Spannungsfeld von fachbezogenen, medien- und grundschulpädagogischen Überlegungen Grundlagen und Erfahrungen für einen kreativen und zugleich reflexiven Einsatz digitaler Medien an Grundschulen erwerben können. In acht Teilprojekten wurden methodische Konzepte entwickelt, die einerseits auf eine Stärkung handlungsorientierter Ansätze abzielten und andererseits die Kooperation zwischen den Fächern und der Medienpädagogik beinhaltete. So wirkten seitens der Fächer die Abteilungen Biologie, Deutsch, Englisch, Musik und Sport sowie das Institut für Mathematik und Informatik mit.

Ausgangspunkt des Projekts waren Hochschulseminare (sog. „Begleitseminare“) an der PH Ludwigsburg, in denen Lehramtsstudierende (vorrangig aus dem Bereich Grundschule) im Rahmen des jeweiligen Teilprojekt-Konzepts Unterrichtseinheiten konzipierten, bei denen die produktive Nutzung digitaler Medien im Grundschulunterricht im Vordergrund stand. Hierbei kamen vorrangig Tablets zum Einsatz, die sowohl für die Hochschule als auch für die Ko-

\footnotetext{
${ }^{2}$ Die folgenden Ausführungen sind angelehnt an einen Beitrag von Horst Niesyto (vgl. Niesyto 2019a), in dem weitere Informationen zur Projektkonzeption nachzulesen sind.

${ }^{3}$ Weitere Informationen zur Schule und zu aktuellen Aktivitäten findet man auf der Webseite der Rosensteinschule: https://rosensteinschule.de/
} 
operationsschule aus Projektmitteln angeschafft wurden. In den jeweiligen Hochschulseminaren gab es in den meisten Fällen eine Tandembetreuung durch Dozent*innen aus den Fachdidaktiken und der Medienpädagogik.

In Form von Unterrichtsversuchen konnten die Studierenden die von ihnen erarbeiteten und vorbereiteten Unterrichtseinheiten mit verschiedenen Grundschulklassen der Rosensteinschule in Stuttgart erproben. Diese Unterrichtsversuche wurden vor Ort von den Lehrpersonen begleitet. In vielen Fällen erfolgte vorab auch ein Austausch über die entwickelten Konzepte. In jedem Teilprojekt gab es eine Pilotphase (mit anschließender Reflexion und teilweise einer Anpassung der Konzeption) sowie eine Durchführungsphase (in der Regel im darauffolgenden Semester). Somit wurden in jedem Teilprojekt jeweils in zwei Semestern bzw. zwei Schulhalbjahren Unterrichtsversuche durchgeführt.

Für die Abteilung Medienpädagogik hatte sich die Mitwirkung an dem Projektprogramm der Deutschen Telekom Stiftung angeboten, weil sich hierdurch die Möglichkeit eröffnete, die bis dato bereits vorgenommenen Aktivitäten zur Implementierung der Medienbildung in der Grundschullehrerbildung (vgl. hierzu Niesyto 2019a, S. 22) in Form eines interdisziplinär ausgerichteten Entwicklungsprojekts zu vertiefen und vor allem die Kooperation mit den Fachdidaktiken in Form von praxisorientierten Seminaren zu intensivieren. Insbesondere für Letzteres wurde bereits im Vorfeld der passende Rahmen geschaffen. Das Entwicklungsprojekt dilegSL bot viele praxisbezogene Erprobungs- und Erfahrungsräume für die beteiligten Akteure an Hochschule und Schule.

In der Projektgruppe wurden die Erfahrungen kontinuierlich reflektiert. Mit Blick auf den Einsatz digitaler Medien in der Grundschule sowie die Gestaltung der Grundschullehrerbildung ging es vor allem darum, Erkenntnisse für eine nachhaltige Verankerung von Medienbildung in der Grundschullehrerbildung zu gewinnen. Hierfür wurden projektbegleitend verschiedene Evaluationsmaßnahmen durchgeführt. Hierzu gehörten neben einer Erhebung von Basisdaten zur Zusammensetzung der beteiligten Schüler*innen und Studierenden (vgl. Niesyto 2019b S. 207f.), einer kriteriengeleiteten Auswertung von zahlreichen Projektjournalen von Studierenden sowie Projektberichten (vgl. Niesyto 2019b), Leitfaden-Interviews mit den mitwirkenden Dozent*innen (fokussierend auf deren Projekterfahrungen; vgl. Junge 2019), ein ausführliches Auswertungsgespräch mit der Schulleitung (nachzulesen in Junge et al. 2019) sowie eine videogestützte Evaluation in Form von Unterrichtsvideografie (vgl. Kürzinger/Pohlmann-Rother 2019).

Die Evaluation des Projekts dileg-SL bezog sich vor allem auf die Akteursgruppe der Studierenden (vgl. Niesyto 2019a, S. 33). In Ergänzung zu den bereits vorliegenden Veröffentlichungen und dem Auswertungsgespräch mit der Schulleitung (Junge et al. 2019) werden in diesem Artikel nun ausgewählte Ergebnisse aus der ebenfalls durchgeführten Lehrenden-Befragung vorgestellt. Mit der Befragung ausgewählter Lehrender, welche die Unterrichtsversuche an unserer Kooperationsschule begleitet haben, sollte auch die Perspektive der Lehrer*innen erfasst werden. Neben projektinternen Fragestellungen (z. B. hinsichtlich der Organisation und den Absprachen untereinander) ging es uns u.a. auch um deren Wahrnehmung bzgl. der mitwirkenden Schüler*innen und Studierenden und deren Einschätzung hinsichtlich der (nachhaltigen) Effekte unserer Projektaktivitäten an der Rosensteinschule. 
Zur Strukturierung der Gespräche wurde vorab ein Leitfaden erstellt. Auf diese Weise konnte unabhängig von der unterschiedlichen Schwerpunktsetzungen der einzelnen Unterrichtsversuche / Teilprojekte eine hinreichende Vergleichbarkeit gewährleistet werden. Die aufgezeichneten Gespräche wurden von einer studentischen Hilfskraft transkribiert und mithilfe der Auswertungssoftware MAXQDA wurden die Aussagen kategorisiert.

Die Leitfaden-Interviews erfolgten während der Projektlaufzeit jeweils nach Abschluss einer Teilprojektphase, also nach der Durchführung der Unterrichtsversuche. Insgesamt wurden fünf Gespräche ${ }^{4}$ mit beteiligten Lehrenden der Rosensteinschule (vier Lehrerinnen, ein Lehrer) durchgeführt und zum Zwecke der näheren Auswertung aufgezeichnet. Sämtliche Gespräche fanden in vertrauter Atmosphäre vor Ort an der Schule statt. Die Aufgabe der Leitfadenerstellung und Gesprächsführung übernahm ich arbeitsteilig zusammen mit Horst Niesyto im Rahmen unserer Rolle als Verantwortliche für die teilprojektübergreifende Evaluation.

Die befragten Lehrenden haben die Unterrichtsversuche der folgenden dileg-Teilprojekte begleitet:

- LK1 (weiblich): Teilprojekt 1 „Intermediales Geschichtenverstehen und Digital Storytelling" (Mitwirkung in der Pilotphase)

- LK2 (männlich): Teilprojekt 1 „Intermediales Geschichtenverstehen und Digital Storytelling" (Durchführungsphase)

- LK3 (weiblich): Teilprojekt 2 „Neue Formen des Lernens - Fächerübergreifender Unterricht mit dem iPad" (Pilot- und Durchführungsphase)

- LK4 (weiblich): Teilprojekt 3 „Trickfilm in der Grundschule“ (Durchführungsphase)

- LK5 (weiblich): Teilprojekt 5 „Gaming im Sportunterricht - virtuelle Bewegungsräume schaffen reale Bewegungsanlässe“ (Durchführungsphase)

\section{Erkenntnisse aus der Lehrenden-Befragung}

Mebrwert der digitalen Medien

Durch die Mitwirkung im Projekt dileg-SL konnten die befragten Lehrenden allgemein die Eignung digitaler Medien für die Grundschule feststellen und konkrete Anwendungsmöglichkeiten kennenlernen. Dabei zeigten sich die befragten Lehrenden, die ja in unterschiedlichen Teilprojekten mitgewirkt hatten, grundsätzlich von der Tauglichkeit der digitalen Medien überzeugt, ohne jedoch eine komplette Abkehr von den traditionellen Lehr- und Lernformen zu befürworten oder anzustreben.

Konkret wurde ein positiver Einfluss auf die Motivation der Schüler*innen genannt, die in der Wahrnehmung der Lehrer*innen durch die digitalen Medien gesteigert werden konnte: „die Motivation der Kinder war sehr hoch, einfach mit den neuen Medien arbeiten zu dürfen“ (LK1 (w) TP1). Es wurde auch berichtet, dass die Mehrzahl der Kinder mit großer Freude bei der Sache war („da waren sie sofort im Fieber“, (LK5 (w) TP5)). Bei einzelnen, ansonsten eher unruhigen Kindern wurde zumindest „phasenweise“(LK4 (w) TP3) eine verbesserte Konzentration beobachtet.

Eine Steigerung der Motivation spielt im Kontext des Tablet-Einsatzes eine wichtige Rolle

\footnotetext{
${ }^{4}$ Aus Zeitgründen konnten leider nicht alle beteiligten Lehrpersonen befragt werden.
} 
und ist auch Gegenstand des mediendidaktischen Diskurses. So hat beispielsweise Stefan Aufenanger, der verschiedene Tablet-Projekte mit Schulen durchgeführt bzw. begleitet hat, positive Effekte wahrgenommen, „die sich u. a. in einer erhöhten Motivation der Schüler*innen beim Lernen sowie einer veränderten Lernkultur im Klassenzimmer ausdrücken “ (Aufenanger 2020a, S. 6). Und die wissenschaftlichen Begleitstudien zum längerfristig angelegten Projekt „Mobiles Lernen in Hessen“ (MOLE) haben gezeigt, dass es sich hierbei nicht um einen kurzfristig Effekt handeln muss: „Ein Nachlassen der Motivation aufgrund eines nachlassenden Neuigkeitseffekts lässt sich dann auch über einen Zeitraum von über zwei Jahren nicht beobachten “(Tillmann 2018, S. 13). Es wird also angenommen, dass eine Motivationssteigerung aufseiten der Schüler*innen nicht ausschließlich einer schnell nachlassenden Neugier am Gerät („Neuigkeitseffekt“) zuzuschreiben ist (vgl. Tillmann/Antony 2018). Zudem wird auf die permanente Entwicklung neuer Applikationen verwiesen, die einem Rückgang des Interesses bzw. der Neugier entgegenwirken kann, sodass die Motivation über einen längeren Zeitraum anhalten kann. Gleichzeitig ist darauf hinzuweisen, dass eine Motivationssteigerung nicht per se gelingt, sondern auch stark von der Gestaltung der Lernszenarien und der Unterrichtsführung abhängt (vgl. Schaumburg 2020, S. 10).

Die (kurzzeitige) Steigerung der Motivation würde ich mit Blick auf unser Projekt allerdings nicht zu stark gewichten. Meines Erachtens lassen sich hieraus keine allgemeinen Schlussfolgerungen mit Blick auf eine langfristige Motivationssteigerung digitaler Medien ziehen. Die Freude der Kinder und ihre große Motivation bzw. ihr Engagement lässt sich sicherlich auf die besondere Situation der Unterrichtsversuche zurückführen. Ich hatte selbst den Eindruck, dass sich die meisten Kinder unabhängig von den mitgebrachten Tablets sehr über den Besuch der Studierenden gefreut haben. Einzelne SuS artikulierten sogar ihre Begeisterung, dass sich jemand so viel Mühe für sie gegeben hat und sie „waren vorher auch sehr aufgeregt“ (LK5 (w) TP5).

Bedingt durch verschiedene Faktoren zeichnet sich die Schülerschaft der Rosensteinschule dadurch aus, dass die Lerngruppen eine hohe Heterogenität aufweisen und viele lernschwache Kinder in den Klassen zu finden sind (vgl. hierzu Aussagen der Schulleitung in Junge et al. 2019, S. 279). Dieser Umstand wurde auch gegenüber den Studierendengruppen kommuniziert und bei der Planung der Unterrichtsversuche berücksichtigt. Und offensichtlich ist es gelungen, mithilfe der digitalen Medien passende Lernszenarien umzusetzen, sodass alle Kinder in gleicher Weise partizipieren konnten. Denn die Integration aller Kinder wurde in sämtlichen Gesprächen als positives Merkmal der jeweiligen Teilprojekte thematisiert. Nach Einschätzung der befragten Lehrenden ermöglichte es der Einsatz der digitalen Medien, alle Kinder einzubinden, unabhängig von ihrem Leistungsvermögen im regulären Fachunterricht. Dies konnte u. a. dadurch gelingen, dass verschiedene Arbeitsschritte ohne eine textbasierte Grundlage oder Verschriftlichung gestaltet wurden. Auf diese Weise konnten sich alle Kinder mit Vorschlägen und Ideen einbringen, bspw. bei der Produktion eines kurzen Video-Clips.

„Dass die Inklusionskinder Teil des Ganzen waren und konnten wirklich mal alles mitmachen gemeinsam mit den anderen Kindern. Das war für mich so prägend, dass es wirklich gut umgesetzt war und das alle teilnebmen konnten." (LK1 (w) TP1)

„Es wurde gut angepasst auch an die Schüler, da ja nicht nur leistungsstarke da dabei sind und manche auch wirklich Probleme haben auch schon alleine beim Lesen, Textverständnis und solchen Dingen. Dass 


\begin{abstract}
man viel auch verbildlicht hat."(LK2 (m) TP1)
„Vor allem, wenn ich jetzt so an die sprachlich schwachen Kinder denke. Die es somit trotzdem ausdrücken konnten, anhand von Bildern, die sie gemacht haben. [...] Die haben wirklich alle ein Produkt erstellen können. [...] Und das hat sie natürlich stolz gemacht oder auch gezeigt sie können etwas leisten. Das war schon ersichtlich. "(LK3 (w) TP2)
\end{abstract}

Somit bestätigten sich unsere im Vorfeld entwickelten Annahmen hinsichtlich der Potentiale einer handlungsorientierten Medienarbeit im Hinblick auf bildungsbenachteiligte Milieus (vgl. hierzu Niesyto/Holzwarth/Maurer 2007; Schluchter 2015; vertiefend zur inklusiven Medienbildung Bosse/Schluchter/Zorn 2019). Es ist gelungen, aus dem Modus der aktiven Produktion mit Medien heraus gestalterische Prozesse anzuregen und mittels medialer Artefakte Ausdrucksmöglichkeiten zu schaffen, die von den Lehrenden als ebensolche wahrgenommen wurden. Allerdings benötigen die betreuenden Lehrenden (in unserem Fall die Lehramtsstudierenden) medienästhetische Kompetenzen, um sich mit den Kindern auch über ästhetische Qualitätsmerkmale auszutauschen (vgl. die teilprojektübergreife Konklusion in Niesyto 2019b). Außerdem hat sich gezeigt, dass es bei Kindern im Grundschulalter gelingen kann, in spielerischer Form (z. B. durch die Verwendung von Scratch) Ansätze zum Computational Thinking zu vermitteln (vgl. hierzu Niesyto 2019b).

Bei einzelnen Kindern, die im regulären Unterricht eher ihre Probleme haben, konnte neben der Freude und dem Spaß ein Lerneffekt konstatiert werden, der auch einen positiven Einfluss auf das Selbstwirksamkeitsempfinden hatte („Und die [Schülerin] konnte sich durch das Tablet-Projekt schon beweisen, dass sie etwas kann.“, (LK3 (w) TP2)). Der Mehrwert ist darin zu sehen, dass im Gegensatz zur klassischen Textarbeit „einfach jeder in der Lage“ (LK2 (m) TP1) war, sich einzubringen und ,auch die gleiche Thematik behandeln konnte“ (LK1 (w) TP1), was im regulären Unterricht aufgrund der sprachlichen Probleme nicht immer möglich ist (vgl. zum Einsatz von Tablets im Deutschunterricht mit sprachlich schwächeren Kindern Lehnert 2018).

Diese Beobachtung im Rahmen des dileg-Projekts deckt sich mit der Einschätzung von Aufenanger, wonach der Einsatz digitaler Medien in der Inklusionspädagogik hilfreich sein kann, weil hierdurch für beeinträchtigte Lernende (alternative) Zugänge zum Unterricht geschaffen werden, deren Realisierung mit analogen Mitteln ungleich aufwendiger wäre. Die Chancen auf Teilhabe können also durch digitale Medien (in unserem Fall vor allem Tablets) gesteigert werden (vgl. Aufenanger 2020a, S. 9), wenn man sie entsprechend einsetzt.

Dieser von den Lehrenden wahrgenommene positive Effekt lässt sich meines Erachtens nicht per se auf den Einsatz digitaler Medien bzw. eine bestimmte, besonders geeignete App zurückführen (vgl. zur Überbewertung digitaler Medien u. a. Irion/Scheiter 2018, S. 8). Vielmehr kommt hierin zum Ausdruck, dass bei der Konzeptionierung auf deren sinnhafte Einbettung in ein methodisch-didaktisches Konzept geachtet wurde, wobei auch die spezifischen Rahmenbedingungen des Unterrichtsversuchs und die Schülervoraussetzungen Beachtung fanden. Dementsprechend wurde bei den Aufgabenstellungen bspw. darauf geachtet, die Arbeitsanweisungen nicht ausschließlich textbasiert zu vermitteln.

$\mathrm{Ob}$ und inwieweit durch dileg-SL eine dauerhafte Förderung visueller, auditiver und audiovisueller Ausdrucksformen durch die produktive Gestaltung von/mit digitalen Medien aufseiten der Schüler*innen realisiert werden konnte, lässt sich meines Erachtens allerdings nicht klar 
benennen. Vielmehr habe ich die Hoffnung, dass den mitwirkenden Lehrenden ein Weg aufgezeigt werden konnte, wie sie sprachlich bzw. textlich weniger begabten Kindern Ausdrucksund Partizipationsmöglichkeiten eröffnen können. Dabei geht es keineswegs um eine Gegenüberstellung oder ein „Ausspielen“ von Text und Bild. Vielmehr sollte ein sinnvolles Zusammenspiel unterschiedlicher Ausdrucksformen (vgl. Konklusion in Niesyto 2019b) angestrebt werden. Wenn Tablets auch zukünftig in dieser Weise eingesetzt werden, kann dies die Förderung eben dieser Kinder unterstützen.

\title{
Medien als Lernwerkzeug kennenlernen
}

Trotz umfassender Forschungsbemühungen liefern die vorliegenden Studienergebnisse bislang keine eindeutigen Erkenntnisse hinsichtlich der Lernwirksamkeit digitaler Medien (vgl. Irion/Scheiter 2018). Wir haben in unserem Projekt auch keinen Schwerpunkt darauf gelegt, mögliche Lerneffekte durch entsprechend aufwendige Messungen zu belegen. Stattdessen war es für uns wichtig, ob und welche Lernzuwächse die am Projekt mitwirkenden Lehrenden durch dileg-SL bei den Kindern beobachten konnten. Die hierzu getätigten Aussagen bezogen sich einerseits auf den Umgang mit den bereitgestellten Tablets und andererseits allgemein auf die Themen/Inhalte der Teilprojekte.

Hinsichtlich der Bedienung der Tablets bescheinigten die befragten Lehrenden den meisten Kindern aus ihrer Klasse umfassende Vorerfahrungen. Diese resultierten nach ihrer Wahrnehmung sowohl aus der privaten Mediennutzung als auch aus den bereits vollzogenen TabletEinsätzen an der Rosensteinschule. Somit zeigten die Kinder überwiegend einen geübten Umgang mit Tablets und kannten sich vereinzelt sogar besser aus als die Lehrenden, was diese jedoch als unproblematisch erachteten. Im Gegenteil wurde darauf verwiesen, dass die Kinder in entsprechenden Situationen ein „Erfolgserlebnis“ (LK4 (w) TP3) haben und sie seien „ja auch stolz, wenn sie was können und was zeigen können“ (LK5 (w) TP5).

Über die bereits vorhandenen Bedienfähigkeiten hinaus konnten viele Schüler*innen ihre kreativen Kompetenzen (,über längere Zeit eine Geschichte auszudenken von Anfang bis Ende“(LK4 (w) TP3)) und medienspezifische Fähigkeiten („wie muss ich wann fotografieren, um den und den Effekt zu erzielen“ (LK2 (m) TP1)) erweitern. Die befragten Lehrenden beschrieben es in diesem Zusammenhang generell als Lernzuwachs aufseiten der Schüler*innen, dass diese durch das Projekt die Tablets auch als Lernwerkzeug erlebt haben. Sie konnten das Tablet einmal auf andere Art und Weise nutzen, im Gegensatz zur unterhaltungsorientierten Anwendung in ihrem privaten Alltag.

\begin{abstract}
„Also YouTube zu öffnen, dass wissen sie. Aber wie man das iPad für den Unterricht nutzen kann oder sinnvoll nutzen kann, da haben sie dann schon einen Lernzuwachs." (LK1 (w) TP1)

„(...) mal eine andere Art und Weise des Umgangs mit dem Tablet, dass es nicht nur zum Spielen da ist, wie man es sonst immer kennt (...)“(LK2 (m) TP1)
\end{abstract}

Wenn die Eindrücke der Lehrkräfte bezüglich der privaten Nutzungsweisen korrekt sind, zeigt sich hieran die Bedeutung der Grundschule hinsichtlich der Vermittlung eines zielführenden produktiven Medienhandelns. Offensichtlich spielt die kreativ-gestalterische Mediennutzung in den Familien der mitwirkenden Kinder eine untergeordnete Rolle. Es wäre wünschenswert, wenn die Grundschule im Zuge der Aktiven Medienarbeit regelmäßig die Potenziale digitaler 
Medien vermittelt und somit auch die Vielfalt dieser digitalen Werkzeuge aufzeigt. Gleichzeitig sollten Lehrende in ihrer Ausbildung sowie im Rahmen der Aus- und Fortbildung dahingehend geschult werden, auch ästhetisch-gestalterische Aspekte zu berücksichtigen (vgl. Niesyto 2019b).

\section{Nachbaltigkeit}

Für die Projektverantwortlichen war es ein wichtiges Ziel, nicht nur kurzfristige Effekte zu erzielen. Da wir dieses Projekt mit einem großen Aufwand betrieben haben, wollten wir einen dauerhaften Beitrag zur Implementierung digitaler Medien an unserer Kooperationsschule leisten. Hierzu wurden vonseiten der Koordinationsgruppe verschiedene Überlegungen angestellt und dokumentiert (vgl. Junge/Niesyto/Rymeš 2019).

Hinsichtlich der nachhaltigen Effekte des Projekts und der dauerhaften Implementierung der im Projekt angeschafften Technik zeigten sich die Befragten keinesfalls euphorisch, aber durchaus zuversichtlich. Sie hatten den Eindruck, dass einzelne Lehrende motiviert werden konnten, sich mit dieser Thematik intensiver auseinanderzusetzen. Allerdings wurde tendenziell angenommen, dass der Prozess einer umfassenden Implementierung digitaler Medien, die sich bspw. in einem regelmäßigen bzw. häufigen Einsatz der angeschafften Tablets ausdrücken könnte, noch mehr Zeit benötigen wird. Von einem schnellen Wandel wurde eher nicht ausgegangen.

Ein wesentlicher Baustein ist die technische Ausstattung an Schulen. Allerdings wird bereits seit vielen Jahren kritisiert, dass die Bedingungen an vielen deutschen Schulen immer noch unzureichend oder zumindest verbesserungswürdig sind (vgl. Ludewig/Knaus/Döring 2013, S. 7), sodass der Einsatz digitaler Medien schon alleine am Fehlen entsprechender Geräte scheitert (vgl. zur Bedeutung der technischen Ausstattung Engel/Knaus/Thülen 2015). Abgesehen von reibungslos funktionierendem W-LAN mangelt es auch an (aktueller) Hardware bzw. mobil einsetzbaren Geräten. Dementsprechend zeigen sich viele Lehrenden sowie Schüler*innen hinsichtlich der verfügbaren Technik unzufrieden (vgl. u. a. WDR-Studie 2019).

Ein erklärtes Ziel des Verbundprojektes „Digitales Lernen Grundschule“ der Deutsche Telekom Stiftung war, diesbezüglich einen Beitrag zu leisten, indem die kooperierenden Schulen auch nach dem Projektende über eine gute technische Ausstattung verfügen. Und so wurde die technische Infrastruktur an der Rosensteinschule im Laufe des Projekts deutlich verbessert. Neben den bereitgestellten Tablets sind nun auch mehrere Klassenräume mit Beamern und Apple-TVs ausgestattet. Diese Verbesserung wird auch von den befragten Lehrenden so wahrgenommen und in den Gesprächen als überaus positive Veränderung artikuliert. Sie äußern sich lobend zum technischen Equipment, welches ihnen nunmehr zur Verfügung steht und sie sehen hierin auch keinen Stolperstein mehr, der einem (regelmäßigen) Einsatz im Wege stehen könnte.

\footnotetext{
„Was definitiv besser geworden ist, ist jetzt ausstattungstechnisch ist die große Anzabl der iPads (...)“(LK2 (m) TP1)

„Also, ich denke Hürden wären eigentlich momentan gut abgebaut [...] liegt schon bisschen auch an einem selbst, da ein bisschen mehr dranzugehen, dann kann man das gut einsetzen, dann kann man das gut machen. [...] Also wir sind hier ja super ausgestattet, man kann sich die Tablets jederzeit im Klassensatz holen. Es ist unkompliziert, ja. Man muss es einfach machen. "(LK4 (w) TP3)
} 
Die Gespräche mit den Lehrenden haben allerdings auch gezeigt, dass Technik lediglich die Basis darstellt. Weitere Hürden wurden nämlich durch die Lehrerfortbildungen abgebaut. Hier hatten die Lehrenden der Kooperationsschule die Möglichkeit, die Tablets und verschiedene Apps kennenzulernen und auszuprobieren (z. T. angeleitet durch unsere Projektmitarbeiter*innen).

Die Bedeutung von Lehrerfortbildungen wird innerhalb des aktuellen Diskurses stark betont (vgl. Bergner 2017; Engel/Knaus/Thülen 2015; Irion/Ruber 2019). Neben einer Verbesserung der technischen Ausstattung (siehe oben) wird hierin ein wesentlicher Schlüssel zur Implementierung digitaler Medien gesehen. Dabei erweisen sich die Strukturen des staatlichen Fortbildungssystems durchaus als Herausforderung (vgl. Eickelmann 2020, S. 41), weshalb es vermutlich zielführend ist, wenn die einzelnen Schulen auf individuelle Maßnahmen wie z. B. Inputs im Rahmen von Lehrerkonferenzen bzw. interne Coaching-Systeme setzen (vgl. Eickelmann 2020, S. 41).

Maßnahmen zur Lehrerfortbildung waren bereits bei der Projektplanung ein wichtiger Baustein des gesamten Verbundprojekts. Und im Projekt dileg-SL entwickelten wir frühzeitig ein grundschulbezogenes Konzept für die Lehrerfort- und -weiterbildung, welches konkret auf die Lehrenden der Rosensteinschule zugeschnitten wurde. Unser vorrangiges Ziel lag darin, die Lehrer*innen mit den Möglichkeiten der Tablets vertraut zu machen und sie zu motivieren, die im Rahmen des Projekts angeschaffte Technik zur Vermittlung von fachlichen und überfachlichen Kompetenzen in ihren Klassen einzusetzen. Hierbei rechneten wir damit, dass zumindest bei einzelnen Teilen des Kollegiums anfangs noch Vorbehalte vorherrschten.

Die Fortbildungsveranstaltungen fanden parallel zu den Teilprojekten statt und adressierten nicht nur die Lehrenden, die in einem Teilprojekt konkret involviert waren. Dementsprechend waren wir um eine hinreichende thematische Vielfalt bemüht und begrenzten uns nicht auf die Inhalte der Teilprojekte. So realisierten wir bspw. eine Fortbildung zum Thema „Sprachförderung mit dem Tablet", an der 12 Personen mitwirkten. Bei der Konzeptionierung weiterer Fortbildungen wurden die Bedürfnisse der Lehrenden berücksichtigt und es zeichnete sich der Wunsch nach einer vertiefenden Auseinandersetzung mit den Tablets als Lehr- und Lernwerkzeug ab.

Um das gesamte Kollegium zu erreichen, waren die ersten beiden Fortbildungsveranstaltungen verpflichtend. Dies war nach Einschätzung der Schulleitung notwendig, damit auch jene „Kolleginnen und Kollegen, die es jetzt nicht so interessiert, [...] dann trotzdem etwas mit(nehmen)“ (Junge et al. 2019, S. 281). Insbesondere mit Blick auf die Lehrenden, die privat kein Tablet besitzen und auch nicht wissen, „wie man eines bedient“ (Junge et al. 2019, S. 282), wurde diese Vorgehensweise rückblickend als „sehr hilfreich“ (Junge et al. 2019, S. 282) erachtet, weil man hierdurch die Grundlagen gelegt hat und „die Hürde heruntergesetzt wurde, um das Tablet wirklich einzusetzen“ (Junge et al. 2019, S. 282).

Grundsätzlich äußerten sich auch die befragten Lehrenden positiv über die Fortbildungen. Nicht zuletzt mit Blick auf die Kolleg*innen, die bislang wenig Berührungspunkte zu digitalen Medien hatten und sich dementsprechend die Bedienung bzw. den Einsatz kaum zutrauten, wurde ein zielführender Effekt wahrgenommen, indem konkrete Anwendungsmöglichkeiten vorgestellt wurden. 
„Ich glaube, wir sind alle sehr glücklich, wenn ein Projekt durchgefübrt wird in unserer Klasse, wir sind aber auch jetzt sehr froh über die Fortbildung. Dass man wirklich mal was an die Hand bekommt, wie kann ich das einsetzen. "(LK1 (w) TP1)

Als besonders wertvoll wurde die Möglichkeit bewertet, die Tablets selbst auszuprobieren. Hierdurch konnten sie offenbar die Distanz zu diesen teils noch ungewohnten Geräten verringern.

"Ich finde es immer ganz wichtig, dass man es auch selbst ausprobieren kann als Lehrer, dass man da wirklich auch ein iPad in die Hand gedrückt bekommt und es ausprobieren kann. Damit man auch weiß, wie man es dann bedient, wenn man selbst dann alleine das machen muss. (lacht)“(LK1 (w) TP1)

Mit den Fortbildungen war die Hoffnung verbunden, dass der Einsatz digitaler Medien auch nach dem Projektende fortgeführt wird und sich im schulischen Alltag etabliert. Ob und inwieweit dies gelungen ist bzw. wie sich der Medieneinsatz möglicherweise zukünftig gestalten wird, war Gegenstand der Lehrenden-Befragungen. Dabei zeigte sich, dass trotz der positiven Bewertung des Projekts und der parallel vollzogenen Lehrerfortbildungen, nicht von einer zügigen und umfassenden Implementierung ausgegangen wird. Vielmehr rechnen die Befragten damit, dass dieser Prozess noch mehr Zeit benötigt. Insgesamt zeigten sich die befragten Lehrenden aber optimistisch, dass die digitalen Medien an ihrer Schule zukünftig häufiger und bei mehreren Kolleg*innen zum Einsatz kommen.

„Durch die Fortbildungen werden eventuelle Hürden, die noch bestehen, immer mebr abgebaut und dann glaube ich, muss man gewissen Dingen bisschen Zeit geben, dass es besser implementiert werden kann. "(LK4 (w) TP3)

"Also, wir sind ja jetzt auch schon drauf und dran, die Kollegen und Kolleginnen immer mebr ins Boot zu bolen."(LK2 (m) TP1)

Direkt nach dem zukünftigen Einsatz der Tablets in den eigenen Klassen befragt, zeigten sich die Befragten durchaus zögerlich. Zwar hatten sie durch die Begleitung der jeweiligen Unterrichtsversuche die Potenziale der Tablets kennengelernt und können auch den Mehrwert digitaler Medien benennen. Aber es scheint mehrere Gründe zu geben, die einer stärkeren / regelmäßigen Nutzung im Weg stehen. So wurden die fehlenden Betreuungsressourcen bzw. allgemein fehlende zeitliche Ressourcen als gravierendes Hindernis benannt. Während die Unterrichtsversuche im Rahmen des Projekts von mehreren Studierenden in Kleingruppen konzipiert und umgesetzt wurden, wobei teilweise sogar noch die Hochschuldozent*innen bzw. studentische Hilfskräfte unterstützend mitwirkten, müssten sie nun allein alles umsetzen. Dies wird skeptisch betrachtet.

„Aber vom Projekt her an sich, äbm, ja, fand ich schwierig, grad so Trailer mit der ganzen Klasse in Kleingruppen umzusetzen, wenn ich nur als eine Lehrerin drin bin. "(LK1 (w) TP1)

„....) ihr Kollege hatte mir auch relativ schnell erklärt, wie man das Tablet als Dokumentenkamera nutzen kann. Das ist was, was ich auch unbedingt umsetzen möchte, sobald ich mir da ein bisschen Raum und Luft dafür geschaffen babe."(LK4 (w) TP3) 
„Es ist natürlich schon noch schwierig. Es klingt alles toll. Es ist auch toll. Die Schule ist auch super ausgestattet mit den Tablets. Ja. Aber gerade alleine im Schulalltag mit den iPads zu arbeiten, mal für eine Stunde oder zwei. Oft hat man die Zeit dann nicht. [...] Aber es ist dann doch oft so, man versucht mit seinem Stoff durchzukommen, dann ist wieder, ja, dann ist immer irgendwas anderes. 1000 Termine. Das ist immer schwierig."(LK5 (w) TP5)

Die Zeitprobleme resultieren auch aus der nicht ganz unkomplizierten Verfügbarkeit der Tablets. Diese befinden sich nämlich im Hauptgebäude, weil sie dort sicher verwahrt und aufgeladen werden können. Außerdem müssen ja sämtliche personenbezogene Daten vor der Benutzung durch den nächsten Kollegen entfernt bzw. extern gesichert werden (vgl. Rymeš/Walter/Iberer 2019). Dies wurde von einer einzelnen Lehrkraft als zu unkomfortabel beschrieben.

\begin{abstract}
„Dann ist es auch oft so, dann muss man die iPads zurückbringen, dann muss man jedes Einzelne wieder genau da anstöpseln, wo es auch war. Du kannst dann nicht einfach das iPad nebmen und schnell den Akku reinmachen. Und das sind natürlich auch alles Ausreden, aber wir sind da alle noch nicht so. Wir sind alle noch ein bisschen bequem."(LK5 (w) TPS)
\end{abstract}

Während der (regelmäßige) Einsatz der Tablets im Unterricht für die Befragten noch mit verschiedenen Fragezeichen verbunden war, wurden mit Blick auf besondere Settings wie den Atelier-Unterricht oder Projektwochen günstige(re) Bedingungen wahrgenommen.

„Also wir haben jetzt ja auch ein Atelier, da wird es genutzt. Wir haben Ateliergruppen, also das ist jabrgangsübergreifender Unterricht zu gewissen Themen und da baben wir jetzt schon zwei Projekte, die mit iPads arbeiten. Also das wird schon eingesetzt. "(LK1 (w) TP1)

„Und in der Medienprojektwoche hatten wir dann auch mit den Tablets [...] diese Puppet-Pals zum Beispiel auch noch mit eingebaut. Das werde ich jetzt auch mit in das Trickfilm-Atelier integrieren "(LK2 (m) TP1)

„Wir haben ja jetzt zum Glück wieder bald die Gesundheitswoche, die ist jetzt im April, die erste Woche nach den Ferien. Da bätte man natürlich die Möglichkeit, die Tablets einzubeziehen. [...] Dann haben wir auch diesen Sporttag. Da kann man auch viel machen, aber jetzt im Unterricht habe ich wie gesagt ja, jetzt noch nichts gezeigt, dass sich da was verändert hat. "(LK5 (w) TP5)

Es wird zwar generell seit längerem diskutiert, den Grundschulunterricht stärker projektorientiert zu gestalten, weil empirische Studien darauf hindeuten, dass hierdurch effektiver gelernt werden könne (vgl. Thissen 2013, S. 28). Und Tablets sind hiermit natürlich gut vereinbar, da sie „die idealen Werkzeuge [sind], die den projektorientierten Unterricht vielfältig unterstützen können“ (ebd.). Aber es ist doch ein wenig enttäuschend, dass die von uns befragten Lehrenden eher diese besondere Form des Lehrens und Lernens mit dem Einsatz von digitalen Medien in Verbindung bringen, anstatt den regelmäßigen Einsatz im regulären Unterricht in den Blick zu nehmen. Letzteres wäre für die Implementierung digitaler Medien von größerem Wert.

Möglicherweise ist dies auch ein Hinweis darauf, dass (noch) mehr (leicht verfügbare) digitale Lernmaterialien vonnöten sind, die sowohl in einer fächerbezogenen als auch in einer fächerübergreifenden Perspektive deutlich zeigen, wie eine aktiv-produktive Gestaltung mit digitalen Medien zielführend in den (alltäglichen) Unterricht integriert werden können.

Es ist zudem bemerkenswert, dass in diesem Zusammenhang auch thematisiert wurde, ob 
und inwieweit sich die Kolleg*innen der Rosenstein-Schule den Einsatz der digitalen Medien nun zutrauen. Während Lehrende durch ihr Studium fundiertes didaktisches Wissen zum Einsatz traditioneller Lernmittel wie Schulbuch, Kreidetafel, Arbeitsblättern und Overheadprojektor besitzen und durch deren Anwendung in der Praxis entsprechende Sicherheit haben, müssen ähnliche Fähigkeiten für die Verwendung digitaler Medien von vielen Lehrenden erst noch erworben werden (vgl. Schaumburg 2020, S. 13). Dies scheint sowohl bei den befragten Lehrenden selbst als auch im Kollegium immer noch ein wichtiger Punkt zu sein. Offensichtlich bedarf es in der Wahrnehmung der Lehrer*innen noch einer gewissen Portion Mut, um dieses Wagnis/Experiment einzugehen. Und hieran scheint es manchen noch zu fehlen.

\begin{abstract}
„Würde ich mir noch nicht zutrauen. Jetzt an sich, zum Beispiel ein Comic zu erstellen (...)“(LK1 (w) TP1) „Und ich glaube schon, dass einige, also die Kollegen sind alle schon interessiert. Wollen auch, aber bei vielen ist es dann einfach auch so, sie trauen sich dann nicht $z u$, zu sagen: $O k$, ich mach jetzt einfach mal diese." (LK2 (m) TP1)

"Natürlich, klar, das ist ein Prozess, vielleicht traut sich da nicht jeder dran, deswegen finde ich es schön, dass wir die Fortbildungen regelmäßig haben, um uns einfach fitter zu machen und dann das den Kindern näher zu bringen. Obwobl die ja oft schon fitter sind."(LK4 (w) TP3)

„(...) klar haben wir hier schon so ein paar Genies, die sich super mit iPads auskennen. Aber ich kenne mich damit auch nicht so gut aus. Es wäre halt toll, wenn man mal zusammen was machen könnte. Zu zweit mit nem Kollegen, dass man sich auch sicherer füblt (...)"(LK5 (w) TP5)
\end{abstract}

Im Rahmen des MOLE-Projekts konnte festgestellt werden, dass die erfahreneren Lehrenden in den Tablet-Klassen mit den technischen Problemen flexibler umgegangen sind und beim Tablet-Einsatz tendenziell mehr gewagt haben als die weniger erfahrenen Lehrenden (vgl. Tillmann 2018, S. 32). Vor diesem Hintergrund kann angenommen werden, dass sich insbesondere junge Lehrer*innen eine gewisse Unbefangenheit bzw. Lockerheit erst aneignen müssen: „Eine nachhaltige Implementierung von digitalen Medien im Unterricht beruht daher auch auf dem Aufbau von Vertrauen, mit unsicheren Situationen umgehen zu können“ (ebd.).

$\mathrm{Ob}$ sich das mangelnde Zutrauen durch eine generelle Unerfahrenheit im schulischen Alltag erklären lässt, kann anhand unserer Befragung nicht klar festgestellt werden. Möglicherweise lässt sich das bislang noch unzureichende Zutrauen dadurch erklären, dass den Lehrenden einfach die Übung fehlt. Eine entsprechende Überlegung formulierte eine Lehrkraft: „Umso bäufiger man es dann gemacht hat, ist es unkompliziert und dann macht man es gern. "(LK4 (w)TP3)

Insbesondere aus dem (weiterhin) geringen Zutrauen in die eigenen Fähigkeiten lassen sich Schlussfolgerungen für Projekte wie dileg-SL ziehen. So kann es sich meines Erachtens als Nachteil erweisen, wenn die mitwirkenden Lehrenden zwar im Vorfeld die Konzeptionen der Studierendengruppen sichten und bei den Umsetzungen der Unterrichtsversuche dabei sind, aber keine konkreten Aufgaben übernehmen. Wenn sie bei der Durchführung zu wenig eingebunden werden, nehmen sie vorrangig die Rolle des begleitenden Beobachters ein. Dies kann zur Folge haben, dass sie auch die Technik nicht bedienen und dementsprechend auch die verwendeten Apps nicht in vertiefender Weise kennenlernen. Nach meiner persönlichen Wahrnehmung war dies bei einzelnen Lehrenden unseres Projekts der Fall. Unabhängig vom Betreu- 
ungs- und Vorbereitungsaufwand stellt sich somit die Frage, ob sie in der Lage wären, die Unterrichtskonzepte, die sie kennengelernt haben, eigenständig umzusetzen.

Im Rahmen unseres Projekts wurde parallel zu den Seminaren für Studierende ein grundschulbezogenes Angebot für die Lehrerfort- und -weiterbildung zum Thema „Medienbildung in der Grundschule“ entwickelt. Während der Projektlaufzeit wurden in Abstimmung mit der Schulleitung und den Lehrer*innen mehrere schulnahe Fortbildungen durchgeführt. Ergänzend wurde der Arbeitskreis „Medienbildung in der Grundschule“ gegründet, der bis heute besteht. Insgesamt haben diese Aktivitäten einen wichtigen Beitrag dazu geleistet, die Lehrenden der Kooperationsschule zu motivieren und zu qualifizieren, digitale Medien in ihrem Unterricht souverän zur Vermittlung von fachlichen und überfachlichen Kompetenzen zu nutzen. Ihre Grenzen finden solche Bemühungen stets im Hinblick auf das Zeitbudget von Lehrenden. Die „Kolleginnen und Kollegen haben wenig Zeit“ (Junge et al. 2019, S. 281) und wenn entsprechende Angebote nicht verpflichtend sind, ist zu beobachten wie „die Bereitschaft zur Teilnahme deutlich abnimmt“ (ebd.). Solange die (zeitliche) Belastung der Lehrenden nicht abnimmt, bleibt dieses Problem leider bestehen und kann Lehrer*innen kaum zum Vorwurf gemacht werden.

Angesichts meiner Eindrücke aus den Unterrichtsversuchen und den Interviews mit ausgewählten Lehrenden bin ich dennoch der Meinung, dass bei praxisorientierten Projekten verstärkt auf eine enge Einbindung der Lehrenden zu achten ist. Im Sinne des Empowerment sollten sie sich von der Rolle des Beobachters lösen und in die Rolle des mitwirkenden Akteurs hineinwachsen. Dies betrifft insbesondere die Bedienung der eingesetzten Medien. Dies kann z. B. dadurch realisiert werden, dass die Lehrenden in Phasen der Gruppenarbeit auch die Betreuung einer Kleingruppe übernehmen (und dies nicht vollständig den Studierenden überlassen) oder die medienspezifischen Arbeitsaufträge (z. B. die Erstellung eines eBooks) ebenfalls durchführen und ihr Ergebnis im Anschluss an die Schüler*innen der Klasse präsentieren. Auf diese Weise würden sie bspw. die Funktionsweise von Tablets nicht nur beobachtend wahrnehmen sondern auch direkt erleben. Gestaltungsmöglichkeiten wie technische Tücken wären auf diese Weise (noch besser) erfahrbar.

Allerdings müssten die hiermit verbundenen (veränderten) Anforderungen im Vorfeld deutlich artikuliert werden, damit die mitwirkenden Lehrenden den damit verbundenen Arbeitsaufwand richtig einschätzen können. Dabei bin ich mir durchaus darüber bewusst, dass dies abschreckend wirken kann und die Unterstützungsbereitschaft von Lehrenden verringern kann. Dessen ungeachtet gehe ich davon aus, dass Lehrkräfte dann mehr aus Projekten „mitnehmen"könnten.

\section{Fazit}

Die Mitwirkung im Projekt dileg-SL war für alle Befragten eine lohnenswerte Erfahrung und die Gesamtbewertung fiel bei allen positiv aus. Die kooperative Zusammenarbeit mit den PHStudierenden und die konkrete Arbeit mit digitalen Medien hat den Lehrer*innen gut gefallen. Außerdem wurde die verbesserte technische Ausstattung an der Rosensteinschule als Gewinn erachtet.

Insgesamt haben uns die Aussagen der befragten Lehrer*innen gezeigt, dass wir mit dileg-SL 
die wesentlichen Projektziele erfolgreich umsetzen konnten. Der Mehrwert, den digitale Medien nach unserer Einschätzung haben, wurde erkannt und wir konnten durch die Unterrichtsversuche deutlich aufzeigen, dass digitale Medien bereits in der Grundschule zielführend eingesetzt werden können. Diesbezüglich kamen in keinem Teilprojekt Zweifel auf. Die positiven Effekte auf die Motivation und die Konzentration der Schüler*innen wurden aufmerksam wahrgenommen und in den abschließenden Interviews uns gegenüber kommuniziert. Für die mitwirkenden Lehrkräfte ist dies sicherlich ein Argument für den wiederholten Einsatz digitaler Medien.

Insbesondere die Bestätigung, dass es gelungen ist, alle Kinder zu integrieren, werte ich als Erfolg. Dies hat uns auch in unserer Entscheidung bestätigt, ungeachtet der besonderen Herausforderungen die Kooperation mit der Rosensteinschule eingegangen zu sein. Mithilfe digitaler Medien können handlungsorientierte Lernszenarien umgesetzt werden, in denen auch jene Kinder Lernerfolge erleben können, die im regulären, textbasierten Unterricht eher benachteiligt sind. Und es bleibt zu hoffen, dass diese Erkenntnis die mitwirkenden Lehrenden bzw. auch das weitere Kollegium motiviert, ähnliche Lernkonzepte zu entwickeln und eigenständig durchzuführen. Es wird auch zukünftig wichtig sein, handlungsorientierte Konzepte zum Medieneinsatz zu entwickeln, die insbesondere Schüler*innen aus den sog. bildungsbenachteiligten Sozialmilieus Räume für Kommunikation, Selbstausdruck, Partizipation und lebensweltnahe Lernprozesse ermöglichen. Dies setzt aber voraus, dass „die vorhandenen lebensund medienweltlichen Erfahrungen der Kinder der Ausgangspunkt für Bildungs- und Lernprozesse sind und digitale Medien in einem vielschichtigen Zusammenspiel von körperlichsinnlichen, wort- und schriftsprachlichen, medial-bildhaften und medial-virtuellen Aneignungs- und Ausdrucksformen genutzt“ (Niesyto 2020, S. 209) werden.

Letztendlich zeigen die Aussagen der mitwirkenden Lehrenden, dass nicht von einer schnellen und umfassenden Implementierung digitaler Medien auszugehen ist, selbst wenn die Anwendungsmöglichkeiten in einem Projektkontext erlebbar waren und die technische Ausstattung einer Schule deutlich verbessert wurde. Auch wenn moderne Technik wie Tablets und entsprechende Peripherie wie Beamer etc. zur Verfügung steht, muss das Zutrauen der Lehrkräfte gestärkt werden, damit diese Technik auch regelmäßig zum Einsatz kommt. Dies bestätigt u. a. Schaumburgs Annahme, wonach es sich bei der Implementierung digitaler Medien in den Unterricht immer noch um ein langwieriges Vorhaben handelt, bei dem die bereits wahrnehmbaren Potenziale erst schrittweise entfaltet werden können (vgl. Schaumburg 2020). Mit Blick auf die eigene Person bzw. den eigenen Unterricht sowie das Kollegium äußerten sich die befragten Lehrenden nicht unbedingt euphorisch, aber langfristig durchaus zuversichtlich.

Ob und inwieweit die Mitwirkung im Projekt dileg-SL dafür gesorgt hat, dass das Kollegium der Rosensteinschule besser mit den Herausforderungen während der Corona-Pandemie zurechtgekommen ist, lässt sich nicht sagen. Meines Erachtens eignen sich die von unseren Studierenden entwickelten Konzepte nur bedingt für den Einsatz im (Notfall-)Fern-Unterricht. Die gemeinsame Bearbeitung einer Arbeitsaufgabe und die räumliche Nähe der Lernenden war in den meisten Fällen ein wesentlicher Bestandteil. Aber möglicherweise resultierte aus unserem Projekt eine größere Affinität zu digitalen (Online-)Medien, was die Umstellung auf Online-Lehre erleichtert hat. Insgesamt habe ich die Hoffnung, dass die schon lange diskutier- 
ten Defizite der schulischen Medienentwicklung, die vor allem, aber nicht nur an der defizitären technischen Ausstattung festzumachen sind, nun endlich in Angriff genommen werden. Wie die Beiträge des Themenschwerpunkts der aktuellen Ausgabe der Ludwigsburger Beiträge zur Medienpädagogik zeigen, liegen viele (positive) Erfahrungen vor, die es nun zu nutzen gilt.

Im Projekt dileg-SL war es uns ein wichtiges Anliegen, dass sich die mitwirkenden Lehramtsstudierenden Kompetenzen für einen pädagogisch-didaktisch sinnvollen Einsatz digitaler Medien (in unserem Fall vor allem Tablets) an Grundschulen aneignen können. Somit fokussierten wir uns z. T. stark auf die kommende Lehrergeneration. Mit Blick auf die Nachhaltigkeit des Projekts an unserer Kooperationsschule haben die Gespräche mit den mitwirkenden Lehrenden deutlich gemacht, welche Nachteile diese Schwerpunktsetzung mit sich bringen kann. Im Sinne eines Empowerments müsste man bei handlungsorientierten Projekten deutlich stärker darauf achten, die Lehrenden aus der Rolle des begleitenden Beobachters zu lösen und zu einem mitwirkenden Akteur zu machen. Denn obwohl sie in den Unterrichtsversuchen den Medieneinsatz „hautnah“ erlebt haben, ist das Zutrauen in die eigenen, mediendidaktischen Fähigkeiten nicht so stark ausgeprägt, wie ich es mir gewünscht hätte. Dementsprechend empfiehlt es sich, sie stärker einzubinden, ihnen die Verantwortung für konkrete Aufgaben zu geben und vor allem mehr Raum für eigene Erfahrungen mit der Technik zu eröffnen. Dann trauen sie sich auch nach Ablauf der Projektzeit eher zu, die digitale Technik eigenständig und ohne fremde Hilfe zu verwenden.

Bereits in der ersten und zweiten Phase der Lehrerbildung müssen solide Grundlagen gelegt werden. Die Forderung nach einer Grundbildung Medien für alle pädagogischen Fachkräfte (vgl. Imort/Niesyto 2014a) ist weiterhin aktuell. An der PH Ludwigsburg wird dies mit dem „Profil Grundbildung Medien“, das 2014 startete und an dem inzwischen 18 Abteilungen, Institute und Einrichtungen der PHL beteiligt sind (vgl. Imort/Niesyto 2014b; Trüby 2017) bereits angegangen. Mit Blick auf die Gestaltung der Lehrerbildung und der Lehrerfortbildung lässt sich aus den Befragungen ableiten, dass die praktische Erprobung von sehr großem Wert ist. Insbesondere in Fortbildungsmaßnahmen sollten anstelle einer ausschweifenden Präsentation über mögliche Einsatzszenarien und Handlungsmöglichkeiten konkrete Anwendungen vorgestellt werden und dann direkt eigenständig ausprobiert werden. Auf diese Weise können sich Lehrende vermutlich ein besseres Bild von einer möglichen Integration in den eigenen Unterricht oder eigene Projekte entwickeln. Schließlich ist es notwendig, dass Lehrende langfristig „anschlussfähig für die gesellschaftlichen Herausforderungen des 21. Jahrhunderts“ (KBoM 2020) sind.

\section{Literatur}

Aktionsrat Bildung (2018): Digitale Souveränität und Bildung. Gutachten. München: Waxmann. URL: www.aktionsrat-bildung.de/fileadmin/Dokumente/Gutachten_pdfs/ARB_Gutachten_Digitale_Souveraenitaet.pdf (01.06.2021)

Aufenanger, Stefan (2020a): Digitale Bildung. In: Aufenanger, Stefan/Eickelmann, Birgit/Feindt, Andreas/Kamin, Anna-Maria (Hrsg.): \#schuleDIGITAL - Friedrich Jahresheft. Kassel: Dierichs Druck, S. 6-9.

Aufenanger, Stefan (2020b): Lehrkräfte sind der Anker gelingender digitaler Bildung. Stefan Aufenan- 
ger im Interview mit Bildungsministerin Stefanie Hubig. In: Aufenanger, Stefan/Eickelmann, Birgit/Feindt, Andreas/Kamin, Anna-Maria (Hrsg.): \#schuleDIGITAL - Friedrich Jahresheft. Kassel: Dierichs Druck, S. 16-17.

Bergner, Nadine (2017): Digitale Bildung in der Schule - die Lehrkräfte sind der Schlüssel. Materialund Fortbildungsangebote zum Thema digitales Lernen. In: MedienPädagogik: Zeitschrift für Theorie und Praxis der Medienbildung (28), S. 123-132. URL: 10.21240/mpaed/28/2017.03.09.X

Bitkom (2019): Lehrer sehen deutsche Schulen digital abgehängt. URL: bitkom.org/Presse/Presseinformation/Lehrer-sehen-deutsche-Schulen-digital-abgehaengt (01.06.2021).

BMBF - Bundesministerium für Bildung und Forschung (2019): Digitalpakt Schule. URL: bildungforschung.digital/de/der-digitalpakt-schule-kommt-2330.html (01.06.2021)

Bosse, Ingo/Schluchter, Jan-René/Zorn, Isabel (2019): Handbuch Inklusion und Medienbildung. Weinheim: Beltz Juventa.

Bundesministerium für Familie, Senioren, Frauen und Jugend (2013): 14. Kinder- und Jugendbericht. Berlin. URL: bmfsfj.de/blob/93146/6358c96a697b0c3527195677c61976cd/14-kinder-und-jugendbericht-data.pdf(01.06.2021).

Eickelmann, Birgit (2020): Zukunftsfähige Schulentwicklung in der digitalisierten Welt. In: Aufenanger, Stefan/Eickelmann, Birgit/Feindt, Andreas/Kamin, Anna-Maria (Hrsg.): \#schuleDIGITAL Friedrich Jahresheft. Kassel: Dierichs Druck, S. 38-41.

Eickelmann, Birgit/Bos, Wilfried/Gerick, Julia/Goldhammer, Frank/Schaumburg, Heike/Schwippert, Knut/Senkbeil, Martin/Vahrenhold, Jan (2019): ICILS-2018 \#Deutschland - Computerund informationsbezogene Kompetenzen von Schülerinnen und Schülern im zweiten internationalen Vergleich und Kompetenzen im Bereich Computational Thinking. Münster: Waxmann.

Engel, Olga/Knaus, Thomas/Thülen, Katharina (2015): Abschlussbericht Projekt fraLine (Oktober 2001 bis August 2014). URL: ftzm.de/images/FTzM/Publikationen/Projektberichte/Abschlussbericht_fraLine4_2015.pdf(01.08.2021).

Grundschulverband (2018): Digitale Mündigkeit beginnt in der Grundschule! Stellungnahme des Grundschulverbands zum „DigitalPakt Schule“ und zum KMK-Beschluss „Bildung in der digitalen Welt“ 2018. URL: grundschulverband.de/wp-content/uploads/2018/08/stellungnahme-gsv-digitalpakt-schule.pdf (01.06.2021)

Imort, Peter/Niesyto, Horst (2014a): Grundbildung Medien in pädagogischen Studiengängen. München: kopaed.

Imort, Peter/Niesyto, Horst (2014b): Editorial zum Themenschwerpunkt: Grundbildung Medien an der PH Ludwigsburg. In: Ludwigsburger Beiträge zur Medienpädagogik, Ausgabe 17/2014, S. 1-3. URL: https://doi.org/10.21240/lbzm/17/01

Irion, Thomas/Scheiter, Katharina (2018): Didaktische Potenziale digitaler Medien in der Grundschule - Der Einsatz digitaler Technologien aus grundschul- und mediendidaktischer Sicht. In: GS aktuell 142, S. 8-11. URL: https://www.pedocs.de/volltexte/2019/16559/pdf/Irion_Scheiter_2018_Didaktische_Potenziale_digitaler_Medien.pdf(01.08.2021).

Irion, Thomas/Ruber, Carina (2019): Das FluX-Prinzip der PH Gmünd. Auf der Suche nach Antworten für die kontextuellen Herausforderungen einer „Digitalen Bildung“ in der Grundschule. In: Junge, Thorsten/Niesyto, Horst (Hrsg.): Digitale Medien in der Grundschullehrerbildung. Erfahrungen aus dem Projekt dileg-SL. Schriftenreihe Medienpädagogik interdisziplinär, Band 12. München: kopaed, S. 367-382.

Junge, Thorsten (2019): Ergebnisse des Entwicklungsprojekts dileg-SL. Eindrücke und Befunde aus den begleitenden Befragungen der Dozenten-Teams. In: Junge, Thorsten/Niesyto, Horst (Hrsg.): Digitale Medien in der Grundschullehrerbildung. Erfahrungen aus dem Projekt dileg-SL. Schriftenreihe Medienpädagogik interdisziplinär, Band 12. München: kopaed, S. 233-262. 
Junge, Thorsten/Niesyto, Horst (2019) (Hrsg.): Digitale Medien in der Grundschullehrerbildung. Erfahrungen aus dem Projekt dileg-SL. Schriftenreihe Medienpädagogik interdisziplinär, Band 12. München: kopaed.

Junge, Thorsten/Niesyto, Horst/Rymeš, Robert (2019): Überlegungen zur Nachhaltigkeit des Entwicklungsprojekts dileg-SL. In: Junge, Thorsten/Niesyto, Horst (Hrsg.): Digitale Medien in der Grundschullehrerbildung. Erfahrungen aus dem Projekt dileg-SL. Schriftenreihe Medienpädagogik interdisziplinär, Band 12. München: kopaed, S. 319-344.

Junge, Thorsten/Niesyto, Horst/Wiemers, Kai/Reichel, Jakob (2019). Das Projekt dileg-SL aus der Perspektive der Schulleitung. In: Junge, Thorsten/Niesyto, Horst (Hrsg.): Digitale Medien in der Grundschullehrerbildung. Erfahrungen aus dem Projekt dileg-SL. Schriftenreihe Medienpädagogik interdisziplinär, Band 12. München: kopaed, S. 277-292.

Kammerl, Rudolf/Dertinger, Andreas/Stephan, Melanie/Thumel, Mareike (2020): Digitale Kompetenzen und Digitale Bildung als Referenzpunkte für Kindheitskonstruktion im Mediatisierungsprozess. In: Thumel, Mareike/Kammerl, Rudolf/Irion, Thomas (Hrsg.): Digitale Bildung im Grundschulalter. Grundsatzfragen zum Primat des Pädagogischen. München: kopaed, S. 21-48.

KBoM (2020): Lernen aus dem Lockdown - die Krise als Chance für Bildung. Positionspapier der Initiative „Keine Bildung ohne Medien!“. URL: www.keine-bildung-ohne-medien.de/wp-content/uploads/2020/10/KBoM_Positionspapier_Corona.pdf (01.06.2021)

Knaus, Thomas (2019): $\mathrm{Pa}(\mathrm{c}) \mathrm{kt}$ Schule digital? - Von Worthülsen und kreativer Schulpolitik. In: b\&w (GEW) Heft 9/2019, S. 14-19. URL: gew-bw.de/index.php?eID=dumpFile\&t=f\&f=85523\&token=7b213ea3fb95ac1c20640f3ed8c7fe9677970942\&sdownload=\&n=bundw-09-19-WEB.pdf (01.08.2021)

Kultusministerkonferenz (2016): Strategie der Kultusministerkonferenz: „Bildung in der digitalen Welt“. URL: kmk.org/fileadmin/Dateien/pdf/PresseUndAktuelles/2018/Digitalstrategie_2017_mit_Weiterbildung.pdf(01.06.2021)

Kürzinger, Anja/Pohlmann-Rother, Sanna (2019): Die videogestützte Evaluation im Projekt dileg-SL. Leitfragen, Ergebnisse und Perspektiven für die Grundschullehrerbildung. In: Junge, Thorsten/Niesyto, Horst (Hrsg.): Digitale Medien in der Grundschullehrerbildung. Erfahrungen aus dem Projekt dileg-SL. Schriftenreihe Medienpädagogik interdisziplinär, Band 12. München: kopaed, S. 263-276.

Lehnert, Judith (2018): Es war einmal ... Digitales Märchentheater im 3. Schuljahr. In: Tillmann, Alexander/Antony, Ingo (2018): Tablet-Klassen. Göttingen: Waxmann.

Ludewig, Yvonne/Knaus, Thomas/Döring, Nicola (2013): Die Wirksamkeit von Medienbildungsinitiativen: Erfolge, Probleme und Lösungsansätze. In: MedienPädagogik: Zeitschrift für Theorie und Praxis der Medienbildung, S. 1-19. URL: doi.org/10.21240/mpaed/00/2013.09.06.X.

Medienpädagogischer Forschungsverbund Südwest (2020): KIM-Studie 2020. Kindheit, Internet, Medien. Basisuntersuchung zum Medienumgang 6- bis 13-Jähriger in Deutschland. URL: www.mpfs.de/studien/kim-studie/2020/ (01.06.2021).

Neuss, Norbert/Spitzer, Manfred (2012): Pro und kontra Mediennutzung. Streitgespräch zwischen Norbert Neuß und Manfred Spitzer. URL: dr-neuss.de/app/download/5789719200/InterviewSpitzer-Neuss.pdf (01.06.2021)

Niesyto, Horst (2019a): Digitales Lernen Grundschule - Ausgangsüberlegungen, Ziele und Strukturen des Entwicklungsprojekts dileg-SL In: Junge, Thorsten/Niesyto, Horst (Hrsg.): Digitale Medien in der Grundschullehrerbildung. Erfahrungen aus dem Projekt dileg-SL. Schriftenreihe Medienpädagogik interdisziplinär, Band 12. München: kopaed, S. 17-37.

Niesyto, Horst (2019b): Ergebnisse des Entwicklungsprojekts dileg-SL. Kernpunkte in teilprojektüber- 
greifender Perspektive. In: Junge, Thorsten/Niesyto, Horst (Hrsg.): Digitale Medien in der Grundschullehrerbildung. Erfahrungen aus dem Projekt dileg-SL. Schriftenreihe Medienpädagogik interdisziplinär, Band 12. München: kopaed, S. 207-232.

Niesyto, Horst (2020): Grundbildung Medien in der Primarstufenbildung. Ergebnisse des Entwicklungsprojekts dileg-SL. In: Thumel, Mareike/Kammerl, Rudolf/Irion, Thomas (Hrsg.): Digitale Bildung im Grundschulalter. Grundsatzfragen zum Primat des Pädagogischen. München: kopaed, S. 191-214.

Niesyto, Horst/Holzwarth, Peter/Maurer, Björn (2007): Interkulturelle Kommunikation mit Foto und Video - Ergebnisse des EU-Projekts CHICAM ,Children in Communication about Migration'. München: kopaed.

Niesyto, Horst/Junge, Thorsten (2020): Digitale Medien in der Grundschullehrerbildung. Erfahrungen aus dem Projekt dileg-SL. In: Rummler, Klaus/Koppel, Ilka/Aßmann, Sandra/Bettinger, Patrick/Wolf, Karsten D. (Hrsg.): Lernen mit und über Medien in einer digitalen Welt (Jahrbuch Medienpädagogik 17), Zeitschrift MedienPädagogik, S. 297-322. URL: doi.org/10.21240/mpaed/jb17/2020.05.12.X

Rymeš, Robert/Walter, Roland/Iberer, Ulrich (2019): Datenschutz beim Einsatz digitaler Medien in der Schule unter Berücksichtigung der rechtlichen Rahmenbedingungen in Baden-Württemberg. Handreichung für Schulleitungen, Lehrerinnen und Lehrer. Pädagogische Hochschule Ludwigsburg. URL: dileg-sl.de/grundschule-datenschutz (01.06.2021)

Schaumburg, Heike (2020): Was wissen wir über digitale Medien im Unterricht? In: Aufenanger, Stefan/Eickelmann, Birgit/Feindt, Andreas/Kamin, Anna-Maria (Hrsg.): \#schuleDIGITAL - Friedrich Jahresheft. Kassel: Dierichs Druck, S. 10-13.

Schluchter, Jan-René (Hrsg.) (2015): Medienbildung als Perspektive für Inklusion - Modelle und Reflexionen für die pädagogische Praxis. München: kopaed.

Thissen, Frank (2013): Mobiles Lernen 3. Eigenverlag. URL: frank-thissen.de/PDF/Mobiles_Lernen_3.pdf (01.06.2021)

Thumel, Mareike/Kammerl, Rudolf/Irion, Thomas (2020) (Hrsg.): Digitale Bildung im Grundschulalter. Grundsatzfragen zum Primat des Pädagogischen. München: kopaed.

Tillmann, Alexander (2018): Begleituntersuchung zum Projekt MOLE - Mobiles Lernen in Hessen. In: Tillmann, Alexander/Antony, Ingo (2018): Tablet-Klassen. Göttingen: Waxmann, S. 13-40.

Tillmann, Alexander/Antony, Ingo (2018): Tablet-Klassen. Göttingen: Waxmann.

Trüby, Daniel (2017): Grundbildung Medien an der PH Ludwigsburg - Erste Zwischenbilanz und Evaluation. In: Ludwigsburger Beiträge zur Medienpädagogik, Ausgabe 19/2017, S. 1-8. DOI: https://doi.org/10.21240/lbzm/19/12

Tulodziecki, Gerhard/Herzig, Bardo/Grafe, Silke (2010): Medienbildung in Schule und Unterricht. Bad Heilbrunn: Klinkhardt.

WDR-Studie (2019): „Drei minus“ für die digitale Ausstattung an deutschen Schulen. Exklusive WDR-Umfrage im Rahmen der ARD-Themenwoche „Zukunft Bildung“. URL: presse.wdr.de/plounge/wdr/programm/2019/11/_pdf/PM_Digitalisierung_an_Schulen.pdf(01.06.2021)

Zylka, Johannes (2018): Digitale Schulentwicklung: das Praxisbuch für Schulleitung und Steuergruppen. Weinheim: Beltz. 


\section{Informationen zum Autor}

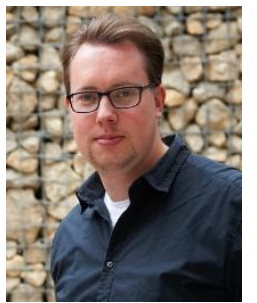

Thorsten Junge arbeitet als Akademischer Oberrat in der Abteilung Medienpädagogik der PH Ludwigsburg. Zu seinen Schwerpunkten gehören die Themen Cybermobbing, Social Media und Digitale Spiele. Seit 2017 ist er in der Redaktion der Ludwigsburger Beiträge zur Medienpädagogik tätig. Er ist Mit-Herausgeber des OnlineSammelbands „Medien im Diskurs“.

thorsten.junge@ph-ludwigsburg.de

\section{Zitationshinweis:}

Junge, Thorsten (2021): Der Einsatz digitaler Medien an der Grundschule - Eindrücke aus dem Projekt dilegSL aus der Perspektive von Lehrkräften. In: Online-Magazin Ludwigsburger Beiträge zur Medienpädagogik, Ausgabe 21/2021. URL: medienpaed-ludwigsburg.de/ 